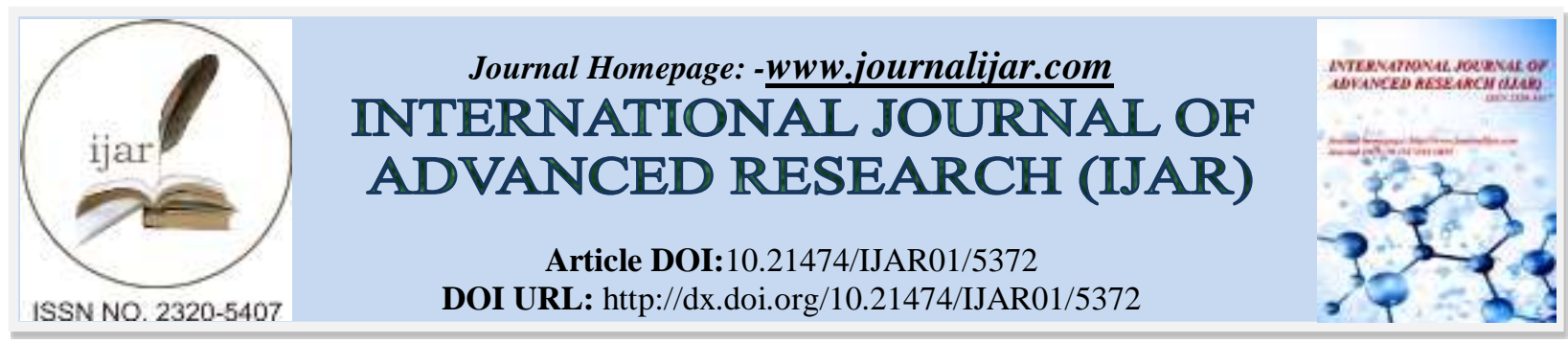

RESEARCH ARTICLE

\title{
STIMULATING QUALITY STANDARD BY BIOMARKERS STANDARDIZATION OF HERBAL FORMULATION FOR DAIRY ANIMALS: PACHOPLUS BOLUS.
}

\author{
Kotagiri Ravikanth, "Deepak Thakur, Anirudh Sharma, Sameer Sapra and Abhinay Chauhan. \\ R\&D Centre, AYURVET LTD, Village Katha, P.O. Baddi - 173205, District Solan, Himachal Pradesh, India.
}

\section{Manuscript Info}

Manuscript History

Received: 09 July 2017

Final Accepted: 11 August 2017

Published: September 2017

Key words:-

Dairy, HPLC, HPTLC, Piperine, Gingerol-6, Pachoplus, Standardization, Validation.

\section{Abstract}

Stupendous increase has been observed in traditional herbal therapy and thus several concerns regarding the safety and quality of herbal medicines have also been observed. There is need for more advanced techniques of standardization. The advancement of analytical techniques will serve as a rapid and specific tool in the herbal research, thereby, allowing the manufacturers to set quality standards and specifications so as to seek marketing approval from regulatory authorities for therapeutic efficacy, safety and shelf- life of herbal drugs. Regulatory authorities ensure that all herbal industrial products subject to their control are in conformity with quality, safety, efficacy that complies with GMP standards. This will improve the quality of the drug and also motivates the practitioners to get more involved in the standardization process. Pharmacological properties of an herbal formulation depend upon phytochemical constituents present therein; development of authentic analytical methods which can reliably profile the phytochemical composition is a major challenge to scientists.Standardization of phytotherapeutic constituents' Gingerol-6 and Piperine with validated analysis methods will help in ensuring the batch to batch consistency in quality \& efficacy of the product on commercial scale.

Copy Right, IJAR, 2017,. All rights reserved.

\section{Introduction:-}

Separation of unique constituents from the herbal mixture is the key step to enable identification and bioactivity evaluation. The chromatography is a powerful analytical method suitable for separation and quantitative determination of a considerable number of compounds, even from a complex matrix. These techniques include High Performance Liquid Chromatography (HPLC) and High Performance Thin-layer Chromatography (HPTLC). These techniques evolve a systematic approach to develop well-designed methodologies for the standardization of herbal raw material and herbal formulations. Standardization is defined as best technical application consensual wisdom inclusive of processes for selection in making appropriate choices for ratification coupled with consistent decisions for maintaining obtained standards. [1-2].

Medicinal plants are the richest bio-resource of traditional drug systems, modern medicines, nutraceutical, food supplements, folk medicines, pharmaceutical intermediates and chemical entities for synthetic drugs. World Health Organization (WHO) encourages, recommends and promotes traditional/herbal remedies in national health care programmes because these drugs are easily available at low cost, safe and people have faith in them. [3-5]. 
Productivity of dairy animals pivots not only on good nutritional diet but also on its proper digestion and assimilation. In ruminants indigestion is very common which generally occurs due to excess ingestion of carbohydrates and sometimes due to nitrogen rich protein diets. The rumen is the most commonly affected, followed by the small intestines. Treatment of animals suffering from primary indigestion in ruminants by conventional drugs is expensive whereas use of herbal drugs, which are cheap, safe and without any side effect can be a good solution. "Pachoplus bolus" a proprietary polyherbal formulation of AYURVET, is one of the preparations used as appetite stimulant and effective in restoration of ruminal micro flora and evacuate the gastrointestinal tract with the intent of eliminating a causative agent. It also facilitates optimal absorption and utilization of nutrients and thus improves feed conversion ratio, productivity and body weight gain. It is a potent herbal formulation, which contains 17 different herbs and minerals. The ingredients of Pachoplus bolus include Terminaliachebula, Allium sativum, Zinziberofficinale, Andrographis peniculata, Trychyspermumammi, Acoruscalamus, Piper nigrum, Piper longumand other. [6-7].

Although individual constituent herbs are scientifically well known to possess appetizer, restorative, carminative, stomachic and tonic activity, standardization of the two pharmacologically prominent herbs i.e Piper nigrum and Piper longum, an appetizer, digestive, carminative, antispasmodic, useful in anorexia, indigestion, constipation, flatulence, diarrhea and Zinziberofficinale for having digestive, antispasmodic activity with respect to the bioactive phyto constituents was taken up to ensure the batch to batch consistency in efficacy. [8].

New HPLC and HPTLC method was developed for the quantification of mentioned herbs with respect to their bioactive marker compounds Gingerol-6 and Piperine an alkaloid. (Figure 1) The analytical method is validated for linearity, accuracy, and precision in accordance with the statistical method of validation given in ICHQ2R1 [9-10]. The average recovery of Gingerol-6 and Piperine was computed from the regression equation. Benchmark limits of two markers are set as quality control check points for batch to batch consistency of formulation in its efficacy.

The method is simple, precise, specific, accurate and has the potential for routine quality control of the formulation.

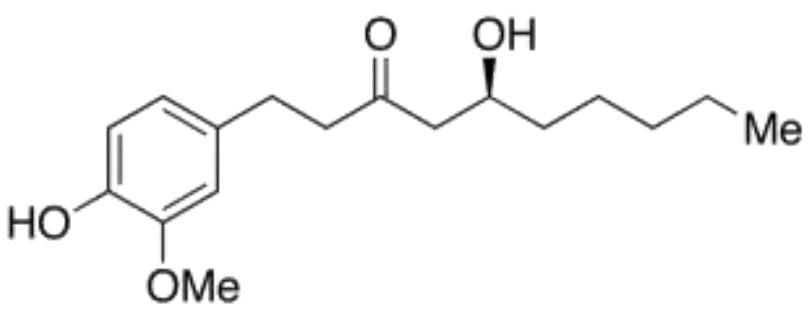

(A)

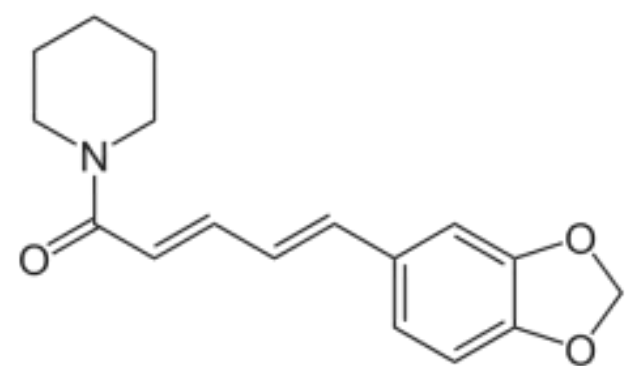

(B)

Figure 1:-Structure of Gingerol-6 (A) and Piperine(B)

\section{Experimental:-}

\subsection{Apparatus}

HPTLC was performed with Camag HPTLC equipment (Muttenz, Switzerland) comprising Linomat-V auto sample applicator, Camag Scanner-III, Camag flat bottom and twin trough developing chamber, and UV cabinet with dual wavelength UV lamp. HPLC was performed with WATERS, USA having binary pump 515 with PDA 2996 detector. The data was acquired on the Empower 2.0 controlling software. Separation was obtained on Phenomenexluna C18 column ( $250 \mathrm{~mm}$ x $4.6 \mathrm{~mm}, 5 \mu \mathrm{m})$.

\subsection{Reagents and materials}

Chemicals and reagents used were of analytical reagent grade. Toluene, diethyl ether, dioxane, acetonitrile and water were purchased from Rankem. Piperine and Gingerol-6 were isolated in house and characterized by different spectroscopic methods before use. TLC plates were purchased from Merck (Darmstadt, Germany). Controlled samples of Pachoplus bolus were obtained from the QA/QC department of AYURVET LTD, Baddi.

\subsection{Chromatographic conditions}

HPLC was performed with WATERS, USA having binary pump 515 with PDA 2996 detector. The data was acquired on the Empower 2.0 controlling software. Separation was obtained on Phenomenexluna C18 column (250 $\mathrm{mm} \times 4.6 \mathrm{~mm}, 5 \mu \mathrm{m})$. The mobile phase was filtered through $0.45 \mu \mathrm{m}$ Millipore filter and degassed. To optimize the 
RP-HPLC parameters, several mobile phase compositions were tried. A satisfactory separation and good peak symmetry for Gingerol-6 [Fig. 1 (A)] was obtained with a mobile phase Acetonitrile : Water : :: $55: 45 \%$ at a flow rate of $1.0 \mathrm{ml} / \mathrm{min}$ to get better reproducibility and repeatability. Selecting $280 \mathrm{~nm}$ as the detection wavelength resulted in acceptable responses and enabled the detection of compound under investigation. HPTLC was performed using commercially-prepared, pre-activated $\left(110^{\circ} \mathrm{C}\right)$ silica gel 60 F254 TLC plates of Merck. A Linomat V (Camag, Muttenz, Switzerland) automatic TLC applicator was used to apply samples and standards (marker compounds) onto the TLC plate under a flow of nitrogen gas. The application parameters were identical for all the analysis performed and the delivery speed of the syringe was $10 \mathrm{~s} / \mu 1$. Each TLC plate was developed to a height of about $8.0 \mathrm{~cm}$, under laboratory conditions with a mobile phase of Toulene : Diethyl ether : Dioxane :: (62.5: $21.5: 16$, v/v/v) for quantification. Quantitative determination of spots corresponding to Fig. 1 (B) was done by Camag TLC Scanner 3 at $260 \mathrm{~nm}$ with a slit size of $6 \times 0.3 \mathrm{~mm}$.

\subsection{Preparation of sample \& standard solutions}

2.4.1 Preparation of standard solutions

Stock solutions ( $0.1 \mathrm{mg} / \mathrm{mL}$ ) of standards (marker compounds) A andBwere prepared in methanol, different concentrations were spotted/injected in order to prepare the calibration graphs and quantification of bioactives.

\subsubsection{Preparation of sample solution for quantification of Gingerol-6 and Piperine}

Weighed accurately around $2 \mathrm{~g}$ of powdered Pachoplus bolus and transferred to a $100 \mathrm{ml}$ round bottom flask. Added $50 \mathrm{ml}$ of methanol, refluxed for 1 hour, repeated the process two more times. Filtered, concentrated and made up the volume to $100 \mathrm{ml}$. Filtered the solution through $0.45 \mu$ syringe filter before using of sophisticated instruments.

\section{Results \&Discussion:-}

The exercise was carried out to ensure the consistency in the desired pharmacological effect by establishing the lowest possible limit for two of its most relevant bioactive phytoconstituents. Standardization of these phytotherapeutic constituents with validated analysis methods will ensure the batch to batch consistency in efficacy of the product on commercial scale. As three herbs mentioned under experimental investigation are among the main active ingredients in the polyherbal formulation, quantifying them with their respective bioactive markers and setting the limits will help us in ensuring authenticity and efficacy of the product in turn.

\section{Method Validation:-}

Validation parameters: The method was validated according to ICH guideline for linearity, precision, accuracy, selectivity, limit of detection and limit of quantification.

4.1 LinearityThe method was validated in accordance with the statistical method of validation given in ICHQ2R1 (30). Two independent calibration equations were obtained. Linear regression analysis was used to calculate the slope, intercept, and coefficient of determination/regression coefficient ( $r 2$ ) for each calibration plot. Response was linear in the concentration ranges investigated (Table 1; Figures $2 \mathrm{f}$ and $3 \mathrm{c}$ ). Evaluation was on the basis of peak area.

a. Calibration: The bio-marker compound in the formulation was quantified using a calibration curve established with five dilutions of the standard at concentrations ranging from and $6-48 \mathrm{ppm}$ for Gingerol-6 standard and 25 $400 \mathrm{ng}$ spot-1 for the Piperine standard compound. The corresponding peak area in formulation was plotted against the concentrations of the standard injected. Peak identification was achieved by comparison of both the Rf/RT and UV absorption spectrum with those obtained for standard.

b. Linearity: Linear regression analysis was used to calculate the slope, intercept, and coefficient of determination/regression coefficient (r2) for calibration plot. Linearity was determined by using five concentrations of the standard solution. The calibration curve was obtained by plotting the area versus the concentrations of the standard solution. Response was linear in the concentration ranges investigated (Fig. 2 f, 3 c, Table 1).

c. Range: Range is the interval between upper and lower concentration (amount) of analyte in sample for which it has been demonstrated that the analytical method has suitable level of precision, accuracy and linearity. The linear response was observed at 280.0nm over a range of $6-48 \mathrm{ppm}$ for Gingerol-6 standard and $340.0 \mathrm{~nm}$ over a range of 25 - 400 ng spot-1 for the Piperine standard (Fig. 2 f, 3 c, Table 1). 


\subsection{Accuracy (\% Recovery)}

Recovery experiments were conducted to check for the presence of positive or negative interferences from other ingredients/excipients present in the formulation and to study the accuracy of the method. Recovery was determined by the standard addition method. Gingerol-6 \&Piperine standards were added to the formulation at two different concentrations, extraction and analysis was performed as described in preparation of sample solution. Recovery was calculated for each standard at each concentration. The results obtained are listed in Table 2.

4.3 Precision Three different concentrations of marker compound solution in triplicates were injected on three different times within the same day and repeating the same on three different days to record intra-day and inter-day variations in the results. The low \%RSD values of Intraday and Interday for marker compound reveals that the proposed method is precise (Table 1).

4.4 Selectivity The selectivity of the respective method was determined by comparing the retention factor/retention time and absorbance spectrum of the standards and the corresponding peaks obtained from the extracts of the formulation. The UV-Vis spectra of both the compounds were compared at three different positions, the peak start, peak center, and peak end. There was good correlation between spectra obtained at each of the three positions. The Gingerol-6 and Piperine peaks separately were, therefore, not masked by any peak of other compound present in the formulation (Figures $2 \mathrm{c}, \mathrm{d}$, e and $3 \mathrm{~d}$ ), which indicated respective peak purity.

4.5 LOD \& LOQ The LOD, defined as the amount of compound required to produce a signal at least three times the noise level. The LOQ, defined as the amount of compound required to produce a signal at least ten times the noise level. The LOD for Gingerol-6 \&Piperine was $0.12 \mathrm{ppm}$ and $0.02 \mu \mathrm{g}$ spot-1 respectively, whereas, the LOQ was $0.36 \mathrm{ppm}$ and $0.06 \mu \mathrm{g}$ spot-1, respectively. Quantification of the amount of Gingerol-6 and Piperine in clinically efficacious batches hence ensures batch to batch reproducibility and consistency in efficacy of the product on commercial scale.

Table 1:-Results of precision, LOD, LOQ, linear regression analysis and their correlation coefficient for quantitative analysis of different marker compounds.

\begin{tabular}{|l|l|l|}
\hline Parameters & Gingerol-6 & Piperine \\
\hline Concentration range & $6-48 \mu \mathrm{g} / \mathrm{ml}$ & $25-400 \mathrm{ng} / \mathrm{spot}$ \\
\hline Regression equation & $\mathrm{y}=1.03 \mathrm{x}+8.07$ & $\mathrm{y}=37.63 \mathrm{x}+1242.77$ \\
\hline Correlation Coefficient $(\mathrm{r} 2)$ & 0.999 & 0.999 \\
\hline Amount of marker compound in Pachoplus bolus [\%] (w/w)a & $0.04 \% \mathrm{w} / \mathrm{w}$ & $0.08 \% \mathrm{w} / \mathrm{w}$ \\
\hline Method precision (Repeatability) - RSD \% & 0.95 & 0.93 \\
\hline $\begin{array}{l}\text { Intermediate precision ( Reproducibility) - RSD \% } \\
\text { Intraday 1 }\end{array}$ & 0.92 & 0.96 \\
Interday 3 & 0.91 & 0.93 \\
\hline LOD & $0.12 \mu \mathrm{g} / \mathrm{ml}$ & $0.02 \mu \mathrm{g} / \mathrm{spot}$ \\
\hline LOQ & $0.36 \mu \mathrm{g} / \mathrm{ml}$ & $0.06 \mu \mathrm{g} / \mathrm{spot}$ \\
\hline
\end{tabular}

Table 2: Results from determination of recovery.

\begin{tabular}{|l|l|l|l|l|l|l|}
\hline Parameter & \multicolumn{3}{l|}{ Gingerol-6 } & \multicolumn{3}{l|}{ Piperine } \\
\hline Initial concentration in formulation [mg g-1] & 0.4 & 0.4 & 0.4 & 0.8 & 0.8 & 0.8 \\
\hline Concentration added [mg g-1] & 0 & 2.0 & 4.0 & 0 & 2.0 & 4.0 \\
\hline Total concentration [mg g-1] & 0.4 & 2.4 & 4.4 & 0.8 & 2.8 & 4.8 \\
\hline Concentration found [mg g-1] & 0.375 & 2.20 & 4.15 & 0.74 & 2.55 & 4.35 \\
\hline RSD [\%] (n=7) & 0.94 & 0.93 & 0.96 & 0.91 & 0.93 & 0.91 \\
\hline Recovery [\%] & 93.75 & 91.67 & 94.32 & 92.5 & 91.07 & 90.63 \\
\hline Mean recovery [\%] & 93.25 & \multicolumn{5}{|l|}{91.40} \\
\hline
\end{tabular}




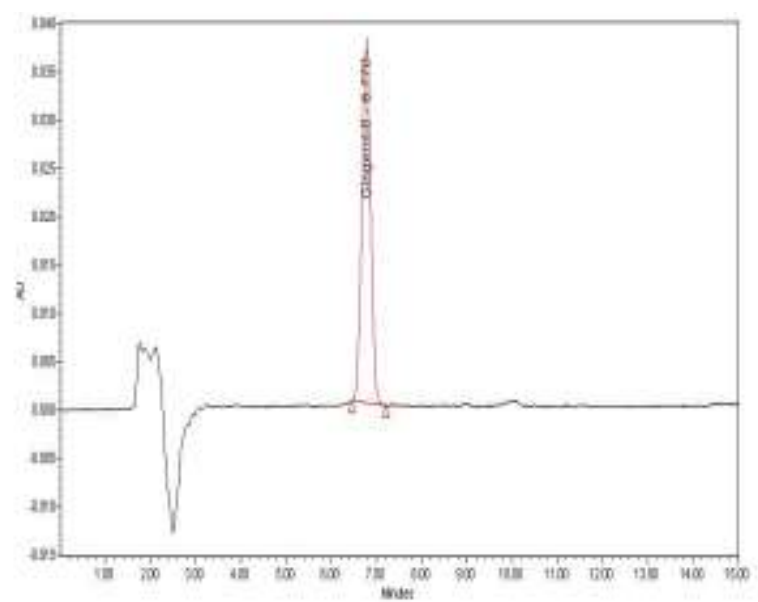

(a)

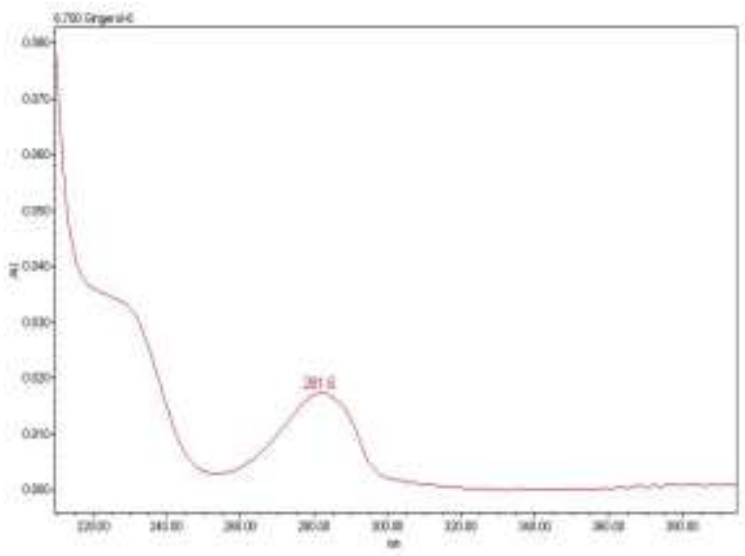

(c)

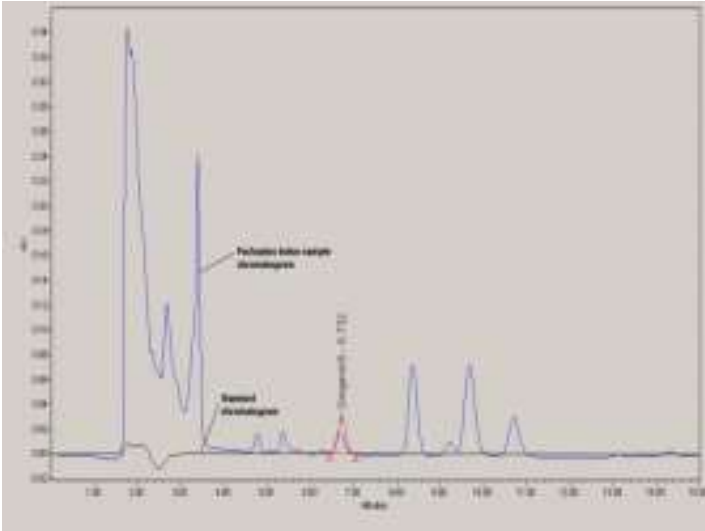

(e)

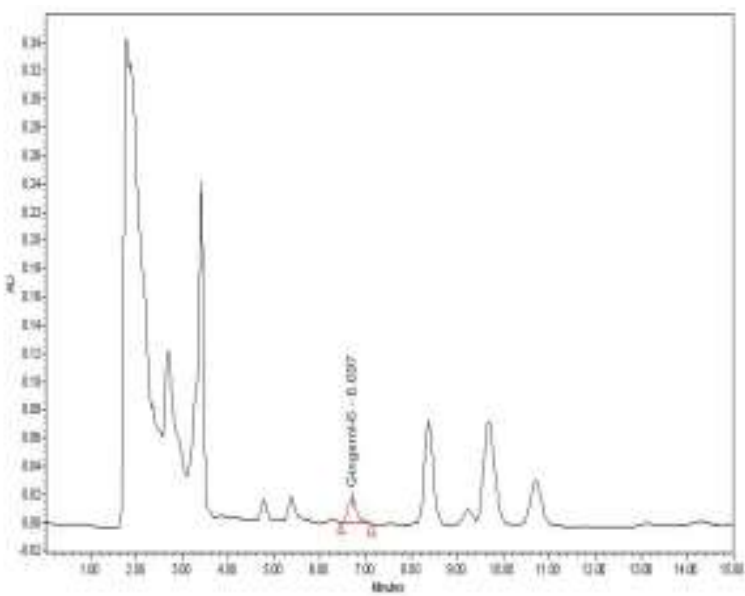

(b)

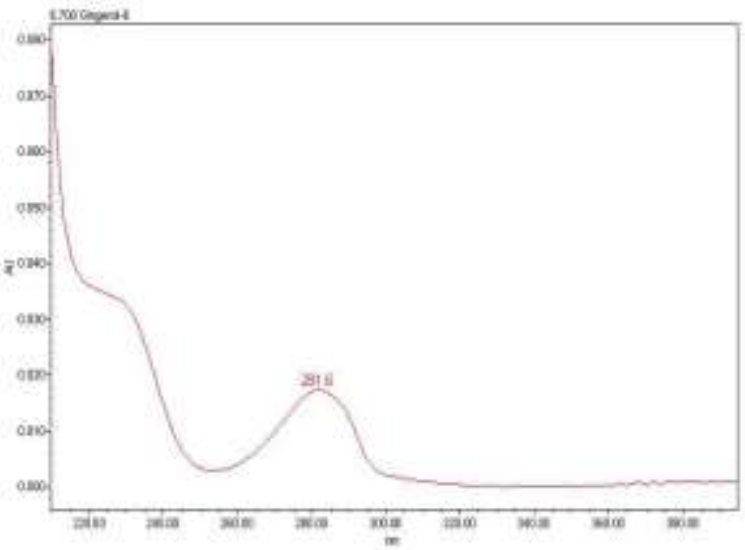

(d)

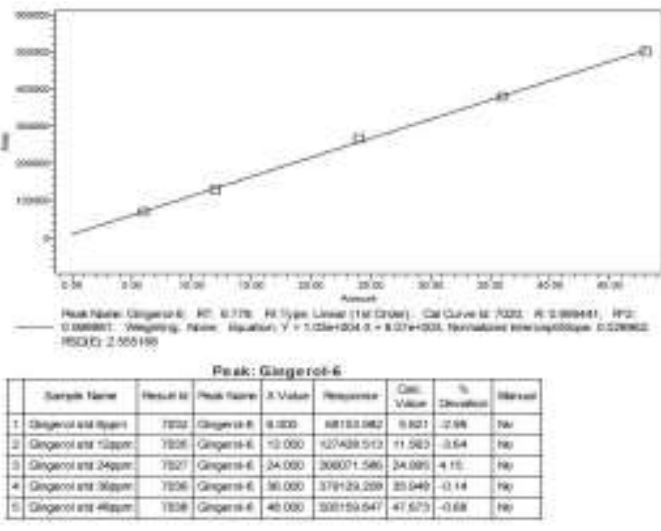

(f)

Figure 2:-Chromatograms showing the resolution of marker compound in the formulation Pachoplus bolus. (a) Chromatogram of the marker compound Gingerol-6. (b) Chromatogram of the formulation Pachoplus bolus.

(c) Chromatogram of spectra of Gingerol-6 standard. (d) Chromatogram of spectra of Gingerol-6 in formulation Pachoplus bolus. (e) Overlay of chromatograms of Gingerol-6 standard with its counterpart in formulation. (f) Calibration plot for Gingerol-6 standard. 


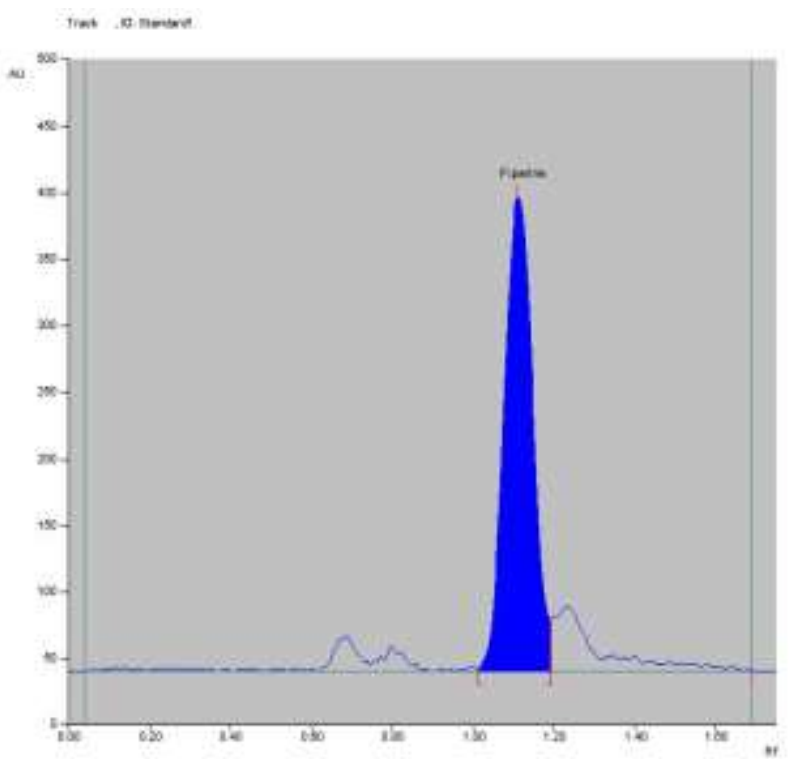

(a)

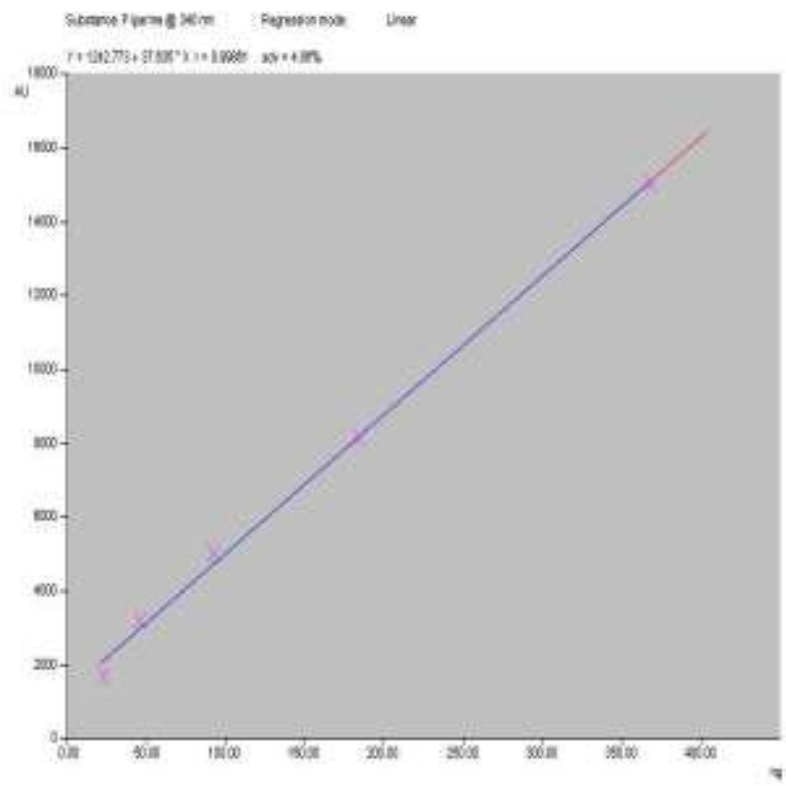

(c)

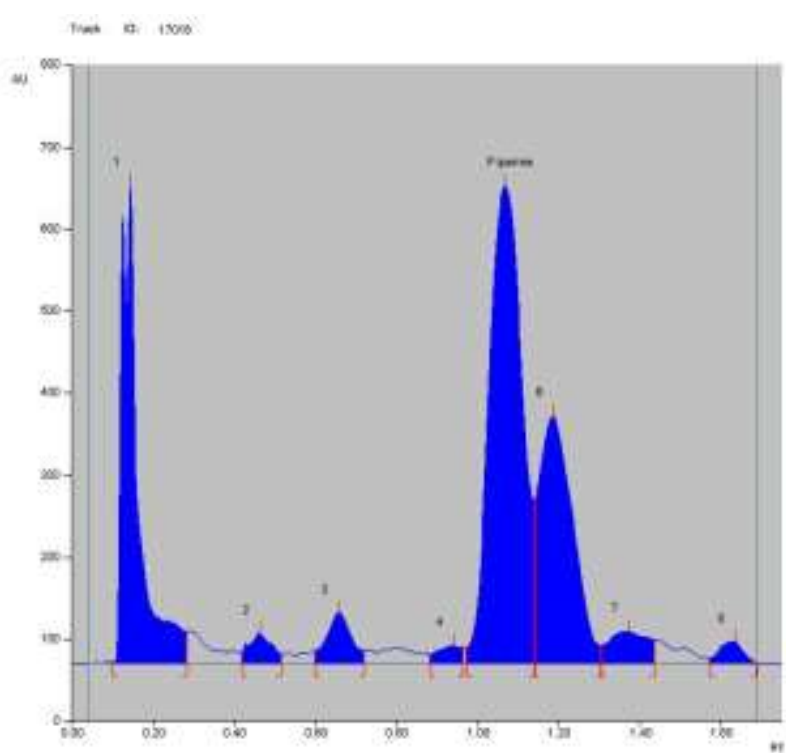

(b)

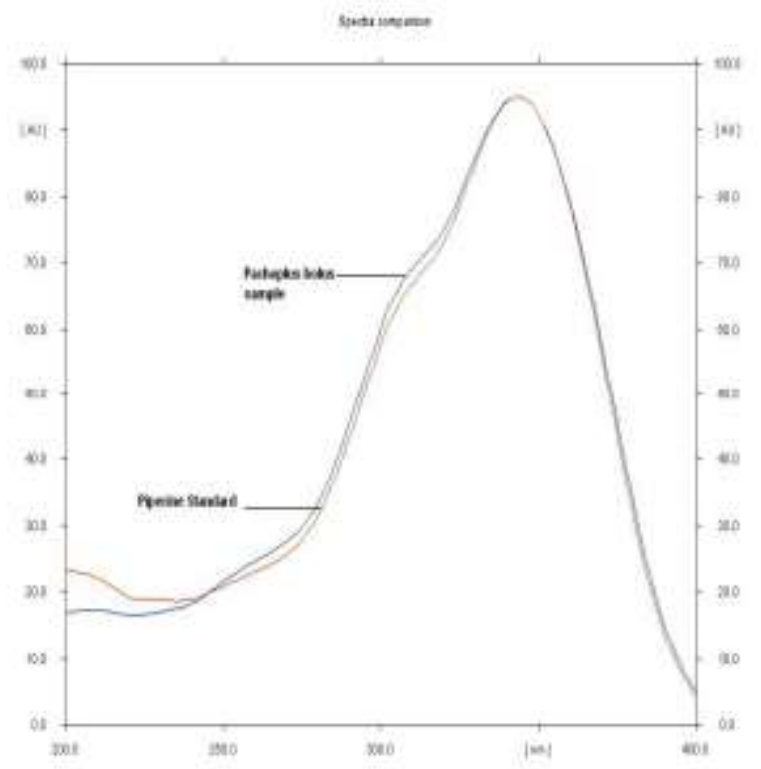

(d)

Figure 3:-Chromatograms showing the resolution of marker compound in the formulation Pachoplus bolus.

(a) Chromatogram of the marker compound Piperine (I). (b) Chromatogram of the formulation Pachoplus bolus. (c) Calibration plot for Piperine standard. (d) Overlay of spectra of Piperine standard with its counterpart in formulation.

\section{Conclusion:-}

The subject of herbal drug standardization is massively wide and deep. New HPLC and HPTLC methods were developed for the fine resolution of two phytoconstituents of the product. Pachoplus bolus, a proprietary polyherbal medicine of Ayurvet Limited for cattle is a complete potent herbal medicine to evacuate the gastrointestinal tract with the intent of eliminating a causative agent and restoration of ruminal micro flora. Standardization of phytotherapeutic constituents' Gingerol-6 and Piperine with validated analysis methods will help in ensuring the batch to batch consistency in quality \& efficacy of the product on commercial scale. Further, methods reported here 
are simple, precise, accurate, there are no signs of any physical or chemical degradation of marker compounds and are suitable for the routine analysis.

\section{Acknowledgement:-}

We thank Mr. MohanjiSaxena, Managing Director, AYURVET Limited, for providing necessary facilities, help and guidance.

\section{References:-}

1. J. B. Harbone, (2005). "Phytochemical Methods-A guide to modern techniques of plant analysis." Springer, New York, NY, USA, 3rd edition.

2. KotagiriRavikanth, Anil Kanaujia, Deepak Thakur et al. (2013) "Validated high performance thin layer chromatographic methods for the quantitative estimation of bioactive constituents in Methiorep premix, a feed additive poly herbal formulation." Journal of planer chromatography. 26 4, 312 - 315 .

3. Ravikanth, K., Kanaujia, A., Thakur, D., Singh, P. (2013) "Bio standardization of an antistresspolyherbal formulation, Stresroak liquid." International journal of pharmacy and pharmaceutical sciences. 5(2), 543 - 546.

4. Ravikanth, K., Thakur, D. et.al. (2016) "Validated analytical methods for the quality standardization of the herbal medicine Janova, an anestrous treatment." International journal of current research, vol. 8, Issue, 12, pp.43085-43091.

5. KotagiriRavikanth, Anil Kanaujia, Deepak Thakur et al. (2014) "Qbs approach for ensuring efficacy of Ruchmax-a polyherbal appetite stimulant and digestive tonic." International journal of pharma sciences. (2014), 4(6) 828- 833.

6. Ashok Kumar and B.P. Joshi. (1996) "A comprehensive study of herbal rumenotorics in buiatrics as alternate therapy". Second plan commonwealth veterinary conference, 319-321.

7. R.K. Bagherwal.(1996) "Therapeutic efficacy of Pachoplus, against indigestion in dairy cattle under field condition.” Indian Veterinary journal. 1996, (3), $254-255$

8. N. Singh, M.A. Akbar, Ramesh Kumari and B.M.Khanna. (1996) "Effect of some treatment of ruminal environment and milk production on clinical case of indigestion in buffaloes." Indian Vet. Med. Jour. Vol. 20, 115-118.

9. ICH Harmonised guidelines, (2005). Q2 (R1), International conference on harmonization, Geneva, 1-13.

10. ICH harmonized tripartite guideline, (2009) Q8 Guidelines for pharmaceutical Development Revision-2, Geneva, Switzerland; 12-14. 